\title{
Euglycemic Diabetic Ketoacidosis Due to Canagliflozin in A Patient with an Uncertain Diagnosis of Type 2 Diabetes: A Case Report
}

\author{
Bonnie B Lu*, Belinda Rivera-Lebron and Jason Ng
}

University of Pittsburgh Medical Center, Pittsburgh, PA, USA

\begin{abstract}
Sodium-glucose cotransport 2 (SGLT2) inhibitors are approved for use only in patients with type 2 diabetes and work by blocking glucose reabsorption in the proximal renal tubule. There is also evidence that SGLT2 inhibitors directly act on pancreatic a-cells to stimulate glucagon secretion, leading to additional ketone body production, and that SGLT2 inhibitors decrease renal clearance of ketone bodies. While the risk of euglycemic diabetic ketoacidosis (eDKA) associated with offlabel use in patients with type 1 diabetes is well known, there are currently no guidelines for SGLT2 inhibitor use in patients with diabetes of uncertain or transitioning pathology. We report a case of eDKA associated with canagliflozin in a patient with rapid progression of noninsulin dependent to insulin-dependent diabetes within the span of 2 years to illustrate the risk of eDKA when SGLT2 inhibitors are used in patients with an uncertain insulin treatment requirement in T2D.
\end{abstract}

Keywords: SGLT2 inhibitor; Euglycemic diabetic ketoacidosis; Noninsulin dependent diabetes; Type 2 diabetes; Canagliflozin; Ketonuria; Metabolic acidosis

\section{Introduction}

Sodium-glucose cotransport 2 (SGLT2) inhibitors were first introduced as a drug class in 2013, and approved for use in patients with Type 2 Diabetes Mellitus (T2D). In the year following approval, there were several reported cases of hospitalization for diabetic ketoacidosis (DKA) with uncharacteristically mild to moderate glucose elevations, also known as euglycemic DKA (eDKA), largely associated with offlabel use in patients with Type 1 Diabetes Mellitus (T1DM) [1]. This led the USA Food and Drug Administration (FDA) to issue a warning regarding the risk of ketoacidosis associated with SGLT2 inhibitor use in March 2015. We report a case of eDKA associated with canagliflozin in a patient with rapid progression of noninsulin dependent to insulindependent diabetes within the span of 2 years to illustrate the risk of eDKA when SGLT2 inhibitors are used in patients with an uncertain insulin treatment requirement in T2D.

\section{Case Report}

A 59-year-old female with non-insulin dependent diabetes presented with one day of nausea, vomiting and severely altered mental status. She was previously diagnosed with T2D 2 years ago. Her most recent $\mathrm{HbAlc}$ was 9.5\%. Her primary care physician was adjusting her outpatient diabetes medication regimen of canagliflozin, dulaglutide, and combination metformin-sitagliptin in the preceding 2 months.

On presentation, she was obtunded with the following: venous $\mathrm{pH}$ of $7.12, \mathrm{pCO}_{2}$ of $13 \mathrm{mmHg}, \mathrm{HCO}_{3}$ of $4 \mathrm{mmol} / \mathrm{L}$, anion gap of 27, and blood glucose of $318 \mathrm{mg} / \mathrm{dL}$. Lactate and kidney function were normal. A complete blood count showed a WBC of $22 \times 10^{3} / \mathrm{uL}$, suggesting possible sepsis. Broad-spectrum antibiotics were started and a bicarbonate infusion was given for acidemia.

Twelve hours from initial presentation, lab work showed persistent acidosis: $\mathrm{pH}$ of 7.26 , serum bicarb of $<5 \mathrm{mg} / \mathrm{dL}$, anion gap of 25 , and blood glucose of 107. A toxic alcohol panel and urine sample showed high levels of acetone $(24 \mathrm{mg} / \mathrm{dL}), 4+$ ketonuria, and glucosuria $(>1000$ $\mathrm{mg} / \mathrm{dL}$ ) with an osmolarity gap of 10 . The toxic alcohol panel and urine comprehensive drug screen were otherwise negative. Antibiotics and bicarbonate infusion were discontinued. She was started on an insulin and dextrose infusion. Over the following 24 hours, her anion gap normalized, $\mathrm{pH}$ improved to 7.44 , and bicarbonate rose to $20 \mathrm{mg} /$ dL. However, repeat urine analysis showed persistent $2+$ ketonuria and glucosuria $(>1000 \mathrm{mg} / \mathrm{dL})$. The insulin and dextrose infusion were continued. After 48 more hours, urinalysis returned negative for ketones, and she was transitioned to a subcutaneous insulin regimen of glargine 12 units at bedtime and lispro 3 units with meals. During her treatment for eDKA, her altered mental status resolved and WBC count normalized. GAD 65 antibodies and islet cell antibodies were negative.

\section{Discussion}

SGLT2 inhibitors work by blocking glucose reabsorption in the proximal renal tubule [2]. Evidence also indicates that SGLT2 inhibitors directly act on pancreatic alpha-cells to stimulate glucagon secretion causing increased ketone body production [3], and that SGLT2 inhibitors decrease renal clearance of ketone bodies [4]. Euglycemic diabetic ketoacidosis (eDKA) has been associated with off-label use in patients with Type 1 Diabetes Mellitus [1]. However, there are currently no guidelines for SGLT2 inhibitor use in patients with diabetes of uncertain or transitioning pathology.

This patient had rapid progression of noninsulin dependent to insulin-dependent diabetes within 2 years. She was of normal weight, had no history of hyperlipidemia or hypertension, or other outward signs of insulin resistance. Her HbA1c of $9.5 \%$ and lack of insulin resistance suggest progressive beta cell failure and loss of insulin production. The patient's GAD 65 and islet cell antibodies were negative. Unfortunately, c-peptide levels and insulin levels were not available to help distinguish between T1D and T2D.

\section{Conclusion}

This case highlights the risk of eDKA when the patient's insulin producing capability is unclear. Certainly, as DM progresses to requiring insulin for treatment, the risk of eDKA would increase. While SGLT2

*Corresponding author: Bonnie B. Lu, University of Pittsburgh Medical Center Pittsburgh, PA, USA, Tel: +1 412-647-2345; E-mail: boshenlu@gmail.com

Received: July 24, 2017; Accepted: August 10, 2017; Published: August 13 2017

Citation: Lu BB, Rivera-Lebron B, Ng J (2017) Euglycemic Diabetic Ketoacidosis Due to Canagliflozin in A Patient with an Uncertain Diagnosis of Type 2 Diabetes: A Case Report. Diabetes Case Rep 2: 127. doi: 10.4172/2572-5629.1000127

Copyright: @ 2017 Lu BB, et al. This is an open-access article distributed under the terms of the Creative Commons Attribution License, which permits unrestricted use, distribution, and reproduction in any medium, provided the original author and source are credited. 
Citation: Lu BB, Rivera-Lebron B, Ng J (2017) Euglycemic Diabetic Ketoacidosis Due to Canagliflozin in A Patient with an Uncertain Diagnosis of Type 2 Diabetes: A Case Report. Diabetes Case Rep 2: 127. doi: 10.4172/2572-5629.1000127

Page 2 of 2

inhibitors are approved for use in T2D, we suggest caution when using in T2D patients with an uncertain insulin treatment requirement status on DM treatment.

\section{Acknowledgment}

The authors thank Holly Filip, PharmD, for assistance in drug literature review.

\section{References}

1. Peters AL, Buschur EO, Buse JB, Cohan P, Diner JC, et al. (2015) Euglycemic diabetic ketoacidosis: A potential complication of treatment with sodiumglucose cotransporter 2 inhibition. Diabetes Care 38: 1687-1693.

2. Ogawa W, Sakaguchi K (2016) Euglycemic diabetic ketoacidosis induced by SGLT2 inhibitors: Possible mechanism and contributing factors. J Diabetes Investig 7: 135-138.

3. Kibbey RG (2015) SGLT-2 inhibition and glucagon: cause for alarm? Trends Endocrinol Metab 26: 337-338.

4. Taylor SI, Blau JE, Rother KI (2015) SGLT2 inhibitors may predispose to ketoacidosis. J Clin Endocrinol Metab 100: 2849-2852. 